\title{
Using emergency physicians' abilities to predict patient admission to decrease admission delay time
}

\author{
Erica E M Lee (1) , ${ }^{1}$ Edmund S H Kwok, ${ }^{1}$ Christian Vaillancourt ${ }^{1,2}$
}

'Department of Emergency Medicine, University of Ottawa, Ottawa, Ontario, Canada ${ }^{2}$ Department of Emergency Medicine, The Ottawa Hospital Research Institute, Ottawa, Ontario, Canada

\section{Correspondence to}

Dr Erica E M Lee, Department of Emergency Medicine, University of Ottawa, Ottawa, Canada; ericlee@toh.ca

Received 24 June 2019 Revised 9 January 2020 Accepted 27 January 2020 Published Online First 5 March 2020

\begin{abstract}
Background In many EDs, emergency physicians (EPs) do not have admitting privileges and must wait for consultants to further assess and admit patients. This delays bed requests and increases ED crowding. We measured EPs' abilities to predict patient admission prior to consultation and estimated the potential ED stretcher time saved if EPs requested a bed with consultation. Methods We conducted a prospective cohort study in an academic centre in Canada between October 2017 and February 2018 using a convenience sample of ED patient encounters requiring consultation. We excluded patients under 18 years or those clearly likely to be admitted (traumas, strokes, S-T elevation myocardial infarctions and Canadian Triage and Acuity Scale of 1). EPs predicted patient admission just before consultation. Potential ED stretcher time saved was estimated for correctly predicted admissions assuming bed requests were initiated with consultation and a constant time to inpatient bed.
\end{abstract}

Results Characteristics of 454 patients were: mean age 60.1 years, $48.5 \%$ male, $46.9 \%$ evening presentation, $69.4 \%$ admitted and median time to bed request of 3.5 hours (IQR 2.0-5.3 hours). Overall, EPs prediction sensitivity, specificity, positive predictive value and negative predictive value were $90.5 \%(95 \% \mathrm{Cl} 86.7 \%$ to $93.5 \%), 84.2 \%$ (95\% Cl $77.0 \%$ to $89.8 \%), 92.8 \%$ (95\% Cl 89.8\% to $95.0 \%)$ and $79.6 \%(95 \% \mathrm{Cl} 73.4 \%$ to $84.7 \%$ ). Approximately 922.1 hours of ED stretcher time could have been saved during the 5-month study period if EPs initiated a bed request with consultation. Conclusion Crowding is a reality for EDs worldwide, and many systems could benefit from EP-initiated hospital admissions to decrease the amount of time admitted patients wait in the ED.

\section{INTRODUCTION}

Patient flow through the ED has been conceptualised using an input-throughput-output model, where system-wide factors influence each transition. ${ }^{1}$ ED crowding occurs when the need for services exceeds what can be provided, and there have been many attempts to address crowding at various points along the model. ${ }^{2}{ }^{3}$ However, the main contributor continues to be access block-the inability of admitted patients to access inpatient wards in a timely matter. ${ }^{245}$

In many academic centres, emergency physicians (EPs) initiate an admission by asking a consultant from the potential admitting service to assess the patient (figure 1). The consultant then assesses the patient, and if they agree that the patient needs admission, initiates the bed request and formal

\section{Key messages}

What is already known on this subject

- In many emergency departments (ED), emergency physicians (EPs) do not have admitting privileges, and must wait for consultants to further assess patients before the admission process can be started

- A previous study found that admitted patients with consultations have substantially longer lengths of stay in the ED than those not receiving consultation

- Prior studies have shown a large variation in the ability of EPs to predict hospital admission

What this study adds

- This prospective cohort study in a Canadian academic hospital found that EPs correctly predicted patient admission in $92.8 \%$ of medical and surgical patients prior to consultation

- If EPs had been able to start the admission process at the same time as requesting a consultation, over 922 hours of ED stretcher time couldhave been saved over a 5-month period

- This suggests that in systems where consultations must precede admission requests, removing this additional step would substantially decrease ED crowding

admission orders. This linear process contributes to the admission delay time, which is composed of the time from consultation to bed request and the time from bed request until an inpatient bed is available. Previous studies have demonstrated that bed request delays make up 33\% of the total time admitted patients spend in the ED. ${ }^{6}$ If admission was initiated by EPs simultaneously with consultation, then theoretically, there would be fewer bed request delays and shorter times to admission.

Previous studies demonstrated EPs can make rapid and accurate disposition decisions. ${ }^{7}$ ED patient flow models and standardised admission protocols have demonstrated that EPs triggering bed requests reduce ED wait times. ${ }^{89}$ Prior studies have measured EPs' disposition predictions at different parts of the patient encounter including at triage, reading the patient's chart without assessment or after a brief assessment ( $<10 \mathrm{~min})$ without any investigations. $^{710-13}$ In those studies, EPs predicted overall disposition with 76.8\%-98.2\% accuracy. However, the studies used a composite outcome of 
Throughput

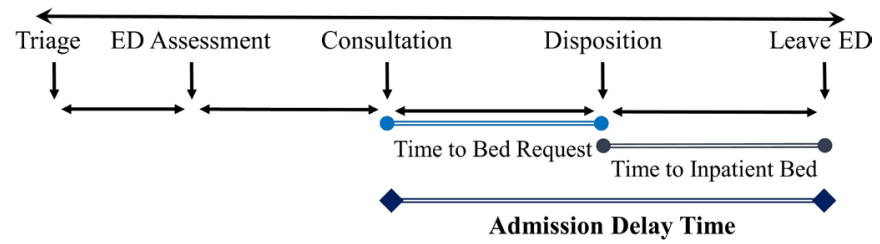

Figure 1 Throughput process of care for patients admitted from the ED. Admission delaytime is composed of the time to bed request, defined as the time from consultation to the time of bed request, and the time to inpatient bed, definedas the time from bed request to exiting the emergency department.

discharge home, transfer or hospital admission. When looking only at admission predictions, EPs' accuracy is lower and more variable (56.4\%-95.8\%).

It is crucial that our consultant colleagues have confidence in the ability of EPs to predict which patients require admission if they are to request a bed prior to consultant assessment. Demonstrating an accurate prediction rate is an important first step in shortening admission delays and substantiating our claim that simultaneous consultation and admission processes would be advantageous for all. Our main objective was to measure the accuracy of EPs admission predictions across various services at the point of requesting a consultation. Our secondary objective was to estimate potential ED stretcher time saved if EPs initiated a bed request at time of consultation.

\section{MATERIALS AND METHODS Study design and setting}

We performed a prospective cohort study at The Ottawa Hospital Civic and General Campuses EDs between October 2017 and February 2018. Both campuses are located in Ottawa, Ontario, Canada, and have a combined annual volume of over $185000 \mathrm{ED}$ visits and an admission rate of 15\%. The hospitals have a total of 1163 acute care beds between both sites. The Civic Campus is a regional Level 1 Trauma Centre and is physically attached to the University of Ottawa Heart Institute; the General Campus is a regional cancer centre. The EDs have 84 treatment bays, including 44 with full bedside monitoring and 2 ambulatory zones. Our ED is staffed by 82 full-time emergency medicine certified attending physicians from the Royal College of Canada and Canadian College of Family Physicians. EPs do not have admitting privileges; only consulting services can admit patients to their service.

\section{Study participants}

Participating EPs were notified by email if they were working during data collection periods and given the opportunity to opt out prior to their shift. As a condition of not requiring written consent for study participation, no identifying EP information was collected. We included ED patient encounters for patients 18 years or older who required at least one consultation. We excluded patient encounters for which an admission to hospital was likely required, including: trauma code, stroke code and S-T elevation myocardial infarction (STEMI) code activations. We also excluded obstetrical emergencies and patients requiring emergent lifesaving care (Canadian Triage and Acuity Scale (CTAS) of 1).

\section{Case identification}

We collected a convenience sample of eligible cases on purposefully selected shifts over a 5 -month period. The principal investigator (EL) was in the ED, floating between care areas where attending EPs would be during their shift. EPs were instructed to notify EL just prior to consultation to give their prediction. In addition, EL was constantly scrutinising the whiteboard feature on the vOACIS electronic medical record interface, which is used to request and track consultations, to ensure no consultations were missed.

\section{Measurements and definitions}

We collected patient characteristics including age and sex, CTAS score, arrival by ambulance and admitting service (if admitted). Time points for arrival, consultation request, bed request and ED departure were collected and used to calculate the time to bed request and time to inpatient bed. Those points, along with other standardised information, were collected on a previously piloted data collection tool.

At the time of consultation, EPs were asked: 'Do you think this patient will be admitted to hospital?'. Their answer was dichotomous: yes or no. If their answer was yes, we assigned a prediction of admission. If they said no, we assigned alternate disposition that included discharge, transfer to another hospital or transfer to a non-acute care facility.

Our standard definition for 'true admission' included: admission to hospital regardless of admitting service, admission recommended but declined by patient (leaving against medical advice), admission for the same disease process within 48 hours of discharge from the ED by the consulting service, patients held overnight without a bed request and if the bed request was delayed by 12 or more hours. We selected admission to hospital to any service as a true admission because the EP was correct in that admission was warranted. We considered representation with admission within 48 hours of initial presentation as true because the patient likely should have been admitted initially. We selected delayed bed request patients as true admission based on Canadian guidelines, which recommend 12 hours as the 90th percentile target for ED length of stay (LOS) for admitted patients. These guidelines suggest that patients should not spend more than 12 hours in the ED waiting for an inpatient bed, thus delays in admission decision beyond that point are equivalent to waiting for admission. ${ }^{2}$ If a patient's final disposition did not meet any of these definitions, then alternate disposition was assigned.

Our proposed admission process would have EPs submit a bed request at the time of consultation. To estimate the 'potential hours of ED stretcher time saved', we used patient encounters with correct admission predictions and created a model with a few assumptions. First, we only included patients for which admission was accurately predicted because these are the only cases where time could have been saved. Second, we assumed the time to inpatient bed did not change. The final assumption was assigning a minimum amount of time that consulting services require to assess the patient in the ED. Our current hospital policy gives services 3 hours to make a disposition decision, so we assumed 3 hours was the minimum amount of time an admitted patient would spend in the ED after a consultation was requested.

Recall that the admission delay time is composed of the time to bed request (time from consultation to initiation of bed request) and the time to inpatient bed (time from bed request until ED exit) (figure 1). To estimate time saved, we first assessed the total 

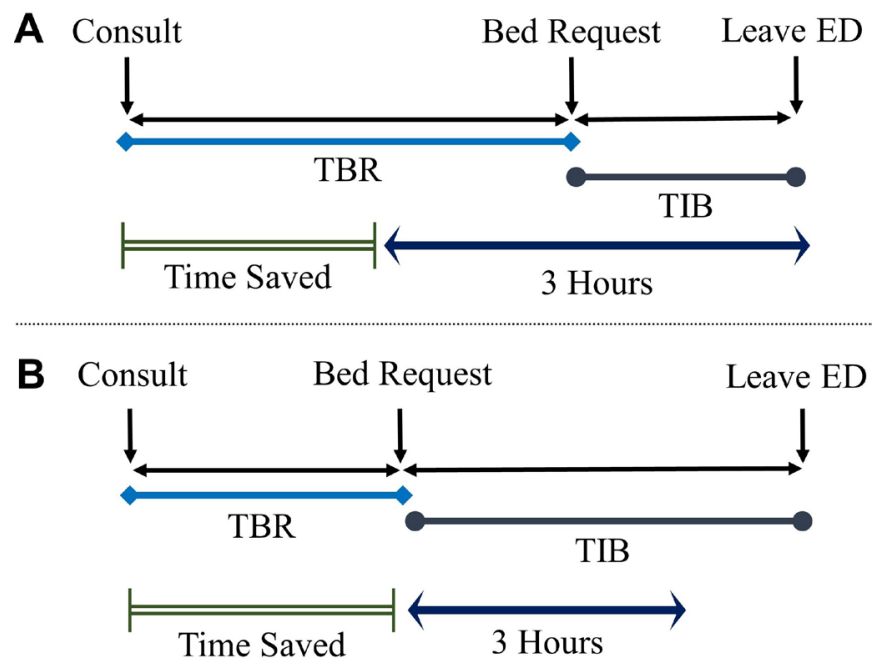

Figure 2 (A) Estimation of ED stretcher time saved where the time to inpatient bed (TIB) is held constant and greater than 3 hours. Time saved is equal to the time to bed request (TBR). (B) Estimation of ED stretcher time saved, where the TIB is held constant and less than 3 hours. Time saved is equal to the TBR less the difference in TIB and minimum consult time of 3 hours.

admission delay time. If it was less than 3 hours, then no time could have been saved as the patients stayed less than the allotted time for a consultant assessment. If the admission delay time was longer than 3 hours and the 'time to inpatient bed' was greater than 3 hours, the time saved is equal to the 'time to bed request' (figure 2A). If the admission delay time was more than 3 hours, but the 'time to inpatient bed' is less than 3 hours, it is the lack of bed request causing delays. Therefore, time saved would be the 'time to bed request' less the difference between the minimum consult time (3 hours) and the 'time to inpatient bed' (figure 2B).

\section{Sample size}

Sample size estimations were calculated according to sensitivity and specificity testing based on previously published data. ${ }^{14}$ We initially attempted to calculate sample size based on work by Steinberg et $a l^{15}$ focusing on the positive predictive value (PPV). Unfortunately, this calculation required more variables than were available; thus, we used sensitivity instead.
We defined prevalence as the percentage of consulted patients that were admitted which, based on two previous Canadian studies, was 55.9\%. ${ }^{6} 16$ We assumed the sensitivity was $90 \%$ based on a previous Canadian study. ${ }^{15}$ We arrived at 424 patients by averaging the sample sizes for 50\% and 60\% prevalence. We wanted to balance campus, shift and day of the week to account for confounding due variations in patient presentations, staffing and patient volume. We separated days of the week into three groups: weekends (Saturday and Sunday), busy weekdays (Monday and Friday) and normal weekdays (Tuesday, Wednesday and Thursday). We chose to collect data on two separate day, evening and night shifts at each campus for each weekday group. Matching these factors required a minimum of 36 shifts, and factoring in our patient volumes and consultation rates, our sample size estimate was 432 .

\section{Statistical analysis}

Simple descriptive statistics using mean with SD or median with IQRs are presented. We calculated EP's ability to predict admission for 11 selected medical and surgical services using sensitivity (percentage of actual admitted patients predicted), specificity (percentage of actual alternate dispositions predicted), PPV (percentage of admission predictions yielding admission) and negative predictive value (NPV) (percentage of alternate dispositions yielding an alternate disposition) with 95\% CIs. We determined the proportion of agreement between EPs and consultant services for admission, alternate disposition and total predictions using Cohen's kappa. Baseline patient characteristics for each prediction were compared via an independent sample t-test for continuous and a $\chi^{2}$ test for categorical variables, where statistical significance was a two-sided $\mathrm{p}$ value $<0.05$. We completed statistical analysis using Vassar Stats online calculator located at http://vassarstats.net/.

\section{RESULTS}

\section{Characteristics of study subjects}

In total, there were 472 consecutive predictions made during the study period accounting for all consultations during sampled shifts. Eighteen patients were excluded as outlined in figure 3, most commonly because the consultation was cancelled $(n=5)$ or moved to an outpatient setting $(n=3)$. Enrolment was stopped after 30 shifts because the sample size had been surpassed.

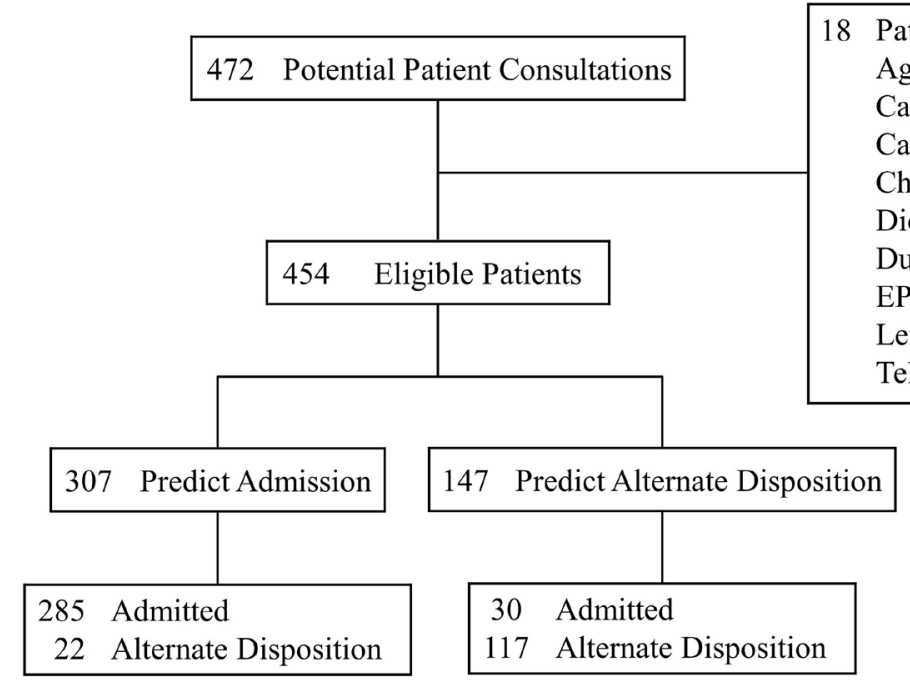

18 Patients Excluded

Age $<18(n=1)$

Cancelled by handover EP $(\mathrm{n}=3)$

Cancelled by original EP $(n=2)$

Changed to outpatient consult $(n=3)$

Died before disposition made $(\mathrm{n}=1)$

Duplicate $(\mathrm{n}=2)$

EP did not make consultation $(n=2)$

Left without being seen by service $(n=2)$

Telephone advice only $(\mathrm{n}=2)$

Figure 3 Flow chart of study enrolment. EP, emergency physician. 
Table 1 Patient characteristics $(n=454)$

\begin{tabular}{|c|c|c|c|}
\hline & $\begin{array}{l}\text { Total } \\
\text { consultations } \\
(\mathrm{n}=454)\end{array}$ & $\begin{array}{l}\text { Admission } \\
(\mathrm{n}=307)\end{array}$ & $\begin{array}{l}\text { Alternative } \\
\text { disposition* } \\
\text { ( } \mathrm{n}=147)\end{array}$ \\
\hline Age, mean, year (range) & $60(18-99)$ & $65(18-99)$ & $51(18-94)$ \\
\hline Male, n (\%) & $220(48.5)$ & $150(48.9)$ & $70(47.6)$ \\
\hline $\begin{array}{l}\text { Arrived by ambulance, } \\
\mathrm{n}(\%)\end{array}$ & $214(47.1)$ & $180(58.6)$ & $34(23.1)$ \\
\hline \multicolumn{4}{|c|}{ Ottawa Hospital Campus, n (\%) } \\
\hline Civic & $228(50.2)$ & $147(47.9)$ & $81(55.1)$ \\
\hline General & $226(49.8)$ & $160(52.1)$ & $66(44.9)$ \\
\hline \multicolumn{4}{|l|}{ Time of consultation, $n(\%)$} \\
\hline Day (08:00-16:00) & $125(27.5)$ & $79(25.7)$ & $46(31.3)$ \\
\hline Evening (16:00-24:00) & $213(46.9)$ & $148(48.2)$ & $65(44.2)$ \\
\hline Overnight (00:00-08:00) & $116(25.6)$ & $80(26.1)$ & $36(24.5)$ \\
\hline \multicolumn{4}{|l|}{ CTAS score, n (\%) } \\
\hline 2 & $204(44.9)$ & $152(49.5)$ & $52(35.4)$ \\
\hline 3 & $231(50.9)$ & $152(49.5)$ & $79(53.7)$ \\
\hline 4 & $18(4.0)$ & $3(1.0)$ & $15(10.2)$ \\
\hline 5 & $1(0.2)$ & $0(0.0)$ & $1(0.7)$ \\
\hline \multicolumn{4}{|l|}{ Time, median, hour (IQR† } \\
\hline $\begin{array}{l}\text { Time to bed request } \\
\text { (TBR)‡ }\end{array}$ & $3.5(2.0-5.3)$ & $3.4(2.1-5.0)$ & $3.6(1.9-6.1)$ \\
\hline $\begin{array}{l}\text { Time to inpatient bed } \\
\text { (TIB)§ }\end{array}$ & $\mathrm{N} / \mathrm{A}$ & $6.1(1.6-15.9)$ & $\mathrm{N} / \mathrm{A}$ \\
\hline Total ED LOSף & $13.4(8.4-21.4)$ & $15.8(10.0-14.6)$ & $8.9(5.8-14.4)$ \\
\hline TBR/ED LOS, mean, \% (SD) & $31.1(20.8)$ & $24.2(16.2)$ & $43.1(22.3)$ \\
\hline TIB/ED LOS, mean, \% (SD) & N/A & $42.3(26.3)$ & N/A \\
\hline
\end{tabular}

*Alternate disposition is composite of any other disposition except admission, including discharge home or transfer to another facility/long-term care.

tSix patients were removed from time data analysis as disposition was entered by EP to transfer to the other site: five from admission predictions and one from alternative disposition.

$\ddagger$ Measured from time consultant service requested until bed request entered.

$\S$ Measured from bed request until ED exit.

IMeasured from time of patient's arrival until the patient exits the department.

CTAS, Canadian Triage Assessment Score; EP, emergency physician; IQR, Interquartile range;

ED LOS, ED length of stay; TBR, time to bed request: TIB, time to inpatient bed.

Characteristics of the 454 included patients were: mean age 60 years with SD of 22 years, $48.5 \%$ male, $46.9 \%$ evening presentation and $69.4 \%$ were admitted (table 1 ). There were no significant differences between admitted and alternate disposition patients with respect to sex, campus or time of consultation. Admitted patients were older, more likely to arrive by ambulance and have CTAS scores of 2. Alternate disposition patients were more likely to have a CTAS score of 4 , but no difference was found between admitted and alternative disposition patients for CTAS scores of 3 . Patients predicted to be admitted had significantly longer ED LOS at 15.8 hours (IQR 10.0-14.6) compared with 8.9 hours (IQR 5.8-14.4).

\section{Primary outcome results}

Overall, EPs predicted admission with sensitivity of 90.5\% (95\% CI $86.7 \%$ to $93.5 \%)$ and a specificity of $84.2 \%$ (95\% CI $77.0 \%$ to $89.8 \%)$. The PPV was $92.8 \%$ (95\% CI $89.8 \%$ to $95.0 \%)$ and NPV was $79.6 \%$ (95\% CI $73.4 \%$ to $84.7 \%$ ). In other words, EPs correctly predicted $92.8 \%$ of patient admissions at the time of consultation. The EPs' disposition predictions by service are summarised in table 2. The PPVs were all above 90\% except for orthopaedic surgery $(84.0 \%$; 95\% CI $63.1 \%$ to $94.7 \%)$ and psychiatry $(78.9 \% ; 95 \%$ CI $53.9 \%$ to $93.0 \%)$. The most commonly consulted service was internal medicine with 122 consultations (26.9\%), which also had the highest PPV at $95.7 \%$ (95\% CI 89.7\% to 98.4\%). Agreement between consultant decision and EP prediction showed kappa values for all dispositions, admission and alternate disposition predictions were 0.885 (95\% CI 0.852 to 0.912 ), 0.846 (95\% CI 0.802 to 0.882 ) and 0.692 (95\% CI 0.616 to 0.760 ), respectively.

There were 52 incorrect predictions of which 22 were incorrect admission predictions. Of these, four were admitted to hospital within 10 days, seven patients asked to be managed as an outpatient and three had bedside procedures completed that facilitated discharge. Of the 30 incorrect alternate disposition predictions, five had new results that changed the diagnosis, three were social admissions because of a deficiency in the community and six were discharged in $<24$ hours.

\section{Secondary outcome results}

There were 13 patient encounters that were removed from the time estimation analysis because the disposition times were entered by the EPs (transfer to other campus for admission) or

Table 2 Emergency physician predictions by consulting services $(\mathrm{N}=454)$

\begin{tabular}{|c|c|c|c|c|c|}
\hline & \multicolumn{5}{|c|}{ Test characteristics, $\%(95 \% \mathrm{Cl})$} \\
\hline & n & Sensitivity & Specificity & PPV & NPV \\
\hline General surgery & 47 & 100.0 (88.0 to 100.0$)$ & 63.6 (31.6 to 87.6) & 90.0 (75.4 to 96.7$)$ & 100.0 (56.1 to 100.0$)$ \\
\hline Gynaecology & 23 & 75.0 (42.8 to 93.3 ) & 90.9 (57.1 to 99.5$)$ & 90.0 (54.1 to 99.5$)$ & 76.9 (46.0 to 93.8$)$ \\
\hline Orthopaedic surgery & 58 & 84.0 (63.1 to 94.7$)$ & $87.9(70.9$ to 96.0$)$ & $84.0(63.1$ to 94.7$)$ & 87.9 (70.9 to 96.0$)$ \\
\hline Other surgical* & 46 & 81.8 (59.0 to 94.0$)$ & 95.8 (76.9 to 99.8$)$ & 94.7 (71.9 to 99.7) & 85.2 (65.4 to 95.1$)$ \\
\hline Intensive care & 3 & 100.0 (31.0 to 100.0$)$ & NDt & 100.0 (31.0 to 100.0$)$ & $\mathrm{ND} \dagger$ \\
\hline Internal medicine & 122 & 96.5 (90.8 to 98.9$)$ & 28.6 (5.1 to 69.7$)$ & 95.7 (89.7 to 98.4$)$ & 33.3 (6.0 to 75.9$)$ \\
\hline Cardiology & 35 & 90.0 (66.9 to 98.2 ) & 86.7 (58.4 to 97.7$)$ & 90.0 (66.9 to 98.2$)$ & 86.7 (58.4 to 97.7 ) \\
\hline Other medicineł & 33 & 85.0 (61.1 to 96.0$)$ & $100.0(71.6$ to 100.0$)$ & $100.0(77.1$ to 100.0$)$ & 81.2 (53.7 to 95.0$)$ \\
\hline Oncological services§ & 27 & 86.9 (65.3 to 96.5$)$ & 75.0 (21.9 to 98.7$)$ & 95.2 (74.1 to 99.7$)$ & 50.0 (13.9 to 86.0$)$ \\
\hline Family medicine & 18 & 100.0 (78.1 to 100.0$)$ & ND† & 100.0 (78.1 to 100.0$)$ & $\mathrm{ND} \dagger$ \\
\hline Psychiatry & 42 & 68.2 (45.1 to 85.3 ) & $80.0(55.7$ to 93.4$)$ & 78.9 (53.9 to 93.0$)$ & 69.5 (47.0 to 85.9 ) \\
\hline Total & 454 & 90.5 (86.7 to 93.5$)$ & 84.2 (77.0 to 89.8$)$ & 92.8 (89.8 to 95.0$)$ & 79.6 (73.4 to 84.7$)$ \\
\hline
\end{tabular}

* Other surgical specialties including otolaryngology, neurosurgery, ophthalmology, oral maxillofacial surgery, plastic surgery, thoracic surgery, trauma surgery, urology and vascular surgery.

tCalculation cannot be performed because of division by zero.

¥0ther medicine services including haematology, gastroenterology, infectious disease, nephrology, neurology and respirology.

§Oncological services include medical, radiation and haematological oncology.

$\mathrm{N}$, total number of patients; $n$, number of patients for that service; ND, not determinable; NPV, negative predictive value; PPV, positive predictive value. 
Table 3 Potentially saved ED stretcher time for admitted patients by service $(\mathrm{N}=272)$

\begin{tabular}{|c|c|c|c|}
\hline & $\mathrm{n}$ & $\begin{array}{l}\text { Total time } \\
\text { saved }^{*} \text {, hour }\end{array}$ & $\begin{array}{l}\text { Time saved per } \\
\text { patient, hour (SD) }\end{array}$ \\
\hline General surgery & 35 & 90.9 & $2.6(2.3)$ \\
\hline Gynaecology & 8 & 23.3 & $2.9(4.8)$ \\
\hline Orthopaedic surgery & 21 & 61.8 & $2.9(3.6)$ \\
\hline Other surgical† & 13 & 27.9 & $2.1(1.7)$ \\
\hline Intensive care & 3 & 7.7 & $2.6(4.4)$ \\
\hline Internal medicine & 108 & 422.9 & $3.9(3.2)$ \\
\hline Cardiology & 18 & 71.4 & $4.0(2.7)$ \\
\hline Other medicine $\ddagger$ & 15 & 48.6 & $3.2(2.0)$ \\
\hline Oncological services§ & 18 & 64.4 & $3.6(2.2)$ \\
\hline Family medicine & 18 & 55.8 & $3.1(1.8)$ \\
\hline Psychiatry & 15 & 47.5 & $3.2(1.4)$ \\
\hline Total & 272 & 922.1 & $3.4(2.8)$ \\
\hline
\end{tabular}

*Calculated assuming the emergency physician initiated a bed request at the time of consultation and the time to inpatient bed was unchanged.

tOther surgical specialties including otolaryngology, neurosurgery, ophthalmology, oral maxillofacial surgery, plastic surgery, thoracic surgery, trauma surgery, urology and vascular surgery.

¥0ther medicine services including haematology, gastroenterology, infectious disease, nephrology, neurology and respirology.

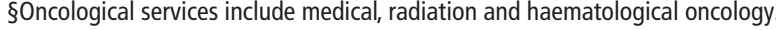
$\mathrm{n}$, number of patients.

the patient did not get a bed request submitted (patients held for $>12$ hours or overnight without a bed request). Approximately 922.1 hours of ED stretcher time could have been saved over the 5-month study period (approximately 30.7 hours per 8-hour shift, 3.4 hours per patient) if EPs initiated a bed request at time of consultation when admission was predicted. Results by consulting services are presented in table 3. For correct admission predictions, it took longer for the service to request a bed than for the patient to get to it in $33.1 \%$ of cases, with the mean bed request delay representing $24.2 \% \pm 16.2 \%$ of their ED LOS.

\section{DISCUSSION}

We completed a prospective cohort study examining EPs' abilities to predict patient admission at the time of consultation. We demonstrated that EPs correctly predicted $92.8 \%$ of patient admissions at the time of consultation for a variety of medical and surgical services in a large tertiary academic centre. EPs and consultant services had high levels of agreement on patients needing admission to hospital with a kappa of 0.846 (95\% CI 0.802 to 0.882 ). We calculated 922.1 hours of ED stretcher time could have been saved during the study period if EPs could initiate a bed request concurrently with consultation when admission was predicted. Admitted patients were older, more likely to arrive by ambulance and have CTAS scores of 2 ; this is not unexpected as admitted patients tend to have more acute illnesses. Patients predicted to be admitted had significantly longer decision delays and ED LOS, which was also anticipated.

Our EPs were able to predict admission at higher rates than previous studies because they had completed their full patient assessment and most of the work-up when their predictions were reported. Previously published studies collected predictions at different points in the patient visit and without the results of any investigations. ${ }^{7}$ 10-13 17 Thus, we expected our EPs to be more accurate because their decisions were more informed.

Predictions for surgical services were slightly less accurate than those for medical services. When reviewing the errors, the most common reasons for surgical consultation were to perform a procedure out of the EP's scope of practice (eg, arthrocentesis of prosthetic joint) or they could not complete themselves (eg, inadequate hernia reduction). In the first situation, EPs did not have results from the definitive diagnostic test (eg, synovial fluid cell counts), which would have influenced their decision. In the second situation, their inability to complete the procedure likely influenced their decision for admission. Furthermore, 'traditional' surgical issues that classically required surgical opinion are now being more aggressively treated in the outpatient setting after a brief period of observation (eg, bowel obstruction that resolves after gastric decompression). Finally, although some surgical procedures can be performed in an urgent outpatient manner, in some cases consultants admitted patients to expedite surgery due to constraints on time or resources that the EP would not be aware of (eg, clinic closed for 2 weeks). Since EPs had to make a prediction prior to consultation, it would be impossible to know this ahead of time. For psychiatry, the PPV was $78.9 \%$, which is similar to previous studies $(66 \%-87 \%){ }^{18-20}$ Reasons for lower prediction abilities include psychiatry's ability to spend more time obtaining collateral history, arrange rapid outpatient follow-up with crisis services not available to EPs and psychiatrists' comfort with discharging suicidal patients.

We placed a higher importance on PPV and sensitivities rather than NPV or specificity because these test characteristics focus on patient admission and we are proposing a change to the admission process. The NPV and specificity are measuring cases when EPs should have initiated a bed request and any time savings would have been lost. Additionally, all patients were seen by consultants who decided their final disposition prior to leaving the ED. At our institution, once the consultant has accepted the patient in consultation, they are now responsible for the patient, so the risk of inappropriately discharging patients (false negative) is borne by the consultants, rather than the EPs.

A consequence of an admission process like this is that EPs are likely to overcall admissions, and consequently there would have been 22 incorrect bed requests during our study period. However, this represents a very small number $(7.4 \%)$ of EP predictions, and it is felt to have a minimal impact because changes to bed requests are common. The admissions office felt that 22 bed cancellations over 5 months would be an insignificant change, creating a single additional bed cancellation per week.

There are some limitations to this study. First, it is a singlecentre Canadian study conducted at an academic centre with a linear admission process, thus the generalisability of our results will be dependent on local practices and healthcare systems. Second, we used convenience sampling of shifts and as such it may not reflect all patient presentations and all EPs ability to make predictions. Third, we removed high acuity patients (STEMI, stroke, CTAS 1 and so on) as including them could have improved our prediction abilities, and finally, there was a risk of contamination bias if the EP spoke with the consulting service prior to recording their prediction. The impact of this was felt to be negligible for two reasons: first, the principal investigator was physically in the ED collecting data in real time and predictions were consistently gathered before discussion with consultants; second, pragmatically, if we adopted a simultaneous bed request and consultation process, discussions between EPs and consulted services would occur.

Based on our calculations, we estimate that patients who were admitted spent approximately 30.7 hours per shift waiting in ED stretchers for consultants' admission decisions. This approximates to 900 hours per month and 10800 hours per year. Over 
a third of admitted patients waited longer for a consultant to initiate a bed request than to access an inpatient bed; waiting represented over $24 \%$ of their ED visit. These long periods of delayed consultant decision making significantly contribute to ED crowding by using ED resources needed to see new patients. This time is what we are hoping to recover by running parallel admission and consultation processes. Internal medicine, which has the most consultations, longest ED stays and highest PPV is the anticipated target for a pilot project exploring a new admission process because it would allow us to gain the most ED stretcher time.

In conclusion, we demonstrated that EPs from a large, tertiary academic centre correctly predicted $92.8 \%$ of patient admissions at the time of consultation and could have saved over 922 hours of ED stretcher time if they initiated a bed request simultaneously. Crowding is a reality for EDs worldwide. While some solutions are either found or imposed, we believe many systems could benefit from EP-initiated hospital admissions to decrease the amount of time admitted patients spend waiting in the ED.

Acknowledgements We would like to thank The Ottawa Hospital emergency physicians, our Ottawa Hospital Research Institute colleague Angela Marcantonio, and Steven Bielby - without their support this project could not have been completed.

Contributors EL and ESHK conceived the study concept. EL recruited participants, performed the data acquisition, data analysis and drafted the manuscript. EK and CV provided advice on study design and data analysis. All authors contributed to its revision and approve of this final version.

Funding The authors have not declared a specific grant for this research from any funding agency in the public, commercial or not-for-profit sectors.

Competing interests None declared.

Patient consent for publication Not required.

Ethics approval This study was approved by the Ottawa Health Science Network Research Ethics Board, which considers the ethical aspects of research studies involving human participants at The Ottawa Hospital.

Provenance and peer review Not commissioned; externally peer reviewed.

Data availability statement Data are available on reasonable request. All data requests will be available on request.

ORCID iD

Erica E M Lee http://orcid.org/0000-0002-3609-0082

\section{REFERENCES}

1 Asplin BR, Magid DJ, Rhodes KV, et al. A conceptual model of emergency department crowding. Ann Emerg Med 2003;42:173-80.

2 Affleck A, Parks P, Drummond A, et al. Emergency department overcrowding and access block. CJEM 2013;15:359-70.

3 Schull MJ, Kiss A, Szalai J-P. The effect of low-complexity patients on emergency department waiting times. Ann Emerg Med 2007;49:257-64.

4 Forster AJ, Stiell I, Wells G, et al. The effect of hospital occupancy on emergency department length of stay and patient disposition. Acad Emerg Med 2003; 10:127-33.

5 Trzeciak S. Emergency department overcrowding in the United States: an emerging threat to patient safety and public health. Emerg Med J 2003;20:402-5.

6 Brick C, Lowes J, Lovstrom L, et al. The impact of consultation on length of stay in tertiary care emergency departments. Emerg Med J 2014;31:134-8.

7 Backay A, Bystrzycki A, Smit DV, et al. Accuracy of rapid disposition by emergency clinicians. Aust Heal Rev 2017;41:133-8.

8 Kang H, Nembhard HB, Rafferty C, et al. Patient flow in the emergency department: a classification and analysis of admission process policies. Ann Emerg Med 2014:64:335-42.

9 Amarasingham R, Swanson TS, Treichler DB, et al. A rapid admission protocol to reduce emergency department boarding times. Qual Saf Heal Care 2010;19:200-4.

10 Wiswell J, Tsao K, Bellolio MF, et al. "Sick" or "not-sick": accuracy of System 1 diagnostic reasoning for the prediction of disposition and acuity in patients presenting to an academic ED. Am J Emerg Med 2013;31:1448-52.

11 Walston JM, Thomas JF, Wiswell JL, et al. 135 How Accurate is "My Gut Feeling?": Comparing the Accuracy of System 1 versus System 2 Decisionmaking for the Acuity Prediction of Patients Presenting to an Emergency Department. Ann Emerg Med 2014;64:S48-9.

12 Vlodaver ZK, Anderson JP, Brown BE, et al. Emergency medicine physicians' ability to predict hospital admission at the time of triage. Am J Emerg Med 2019;37:478-81.

13 Vaghasiya MR, Murphy M, O'Flynn D, et al. The emergency department prediction of disposition (EPOD) study. Australas Emerg Nurs J 2014;17:161-6.

14 Bujang MA, Adnan TH. Requirements for minimum sample size for sensitivity and specificity analysis. J Clin Diagnostic Res 2016;10:YE01-6.

15 Steinberg DM, Fine J, Chappell R. Sample size for positive and negative predictive value in diagnostic research using case-control designs. Biostatistics 2008;10:94-105.

16 Woods RA, Lee R, Ospina MB, et al. Consultation outcomes in the emergency department: exploring rates and complexity. CJEM 2008;10:25-31.

17 Dent AW, Weiland TJ, Vallender L, et al. Can medical admission and length of stay be accurately predicted by emergency staff, patients or relatives? Australian Health Review 2007:31:633-41.

18 Douglass AM, Luo J, Baraff L. Emergency medicine and psychiatry agreement on diagnosis and disposition of emergency department patients with behavioral emergencies. Acad Emerg Med 2011;18:368-73.

19 Chakravarthy B, Menchine M, Thompson DE, et al. Psychiatric patient disposition agreement between the emergency physician and the psychiatry consultant. Crisis 2013:34:354-62.

20 Drescher MJ, Russell FM, Pappas M, et al. Can emergency medicine practitioners predict disposition of psychiatric patients based on a brief medical evaluation? Eur $\int$ Emerg Med 2015;22:188-91. 